\title{
SPECTRAL CHARACTERISTICS OF A CORONAL OSCILLATION WITH A PERIOD OF THE ORDER OF $10 \mathrm{MS}$
}

\author{
XIA ZHIGUO, CHEN JINGYING, ZHENG XIANGMIN \\ AND MA YUAN \\ Yunnan Observatory, Chinese Academy of Sciences, China
}

\begin{abstract}
A coronal oscillation with a changing period was observed with the acousto-optic radio spectrometer of Yunnan Observatory. The bandwidth of the oscillation was about $10 \mathrm{MHz}$. It accompanied radiation which lasted for about $500 \mathrm{~ms}$. A corresponding burst was found on a radio spectrogram obtained at Weissenau, Germany. A 245 $\mathrm{MHz}$ burst was reported at the same time and we found a bright spot on the $\mathrm{H} \alpha$ film records of Yunnan Observatory. In this paper, we present a series of spectra of the oscillation at $10 \mathrm{~ms}$ intervals. In the spectra the oscillation appears in the form of a $3.5 \mathrm{MHz}$ drift of radio flux with frequency.
\end{abstract}

\section{INTRODUCTION AND OBSERVATIONS}

On 1990 August 23, during the 15th joint observation period for Cycle 22 in China, we observed varying-period radio oscillations with a 10-meter antenna and a high-resolution acousto-optic radio spectrometer (AOS). The time and frequency resolution of the AOS are $10 \mathrm{~ms}$ and $0.5 \mathrm{MHz}$. These characteristics give us good observational capabilities for observing the frequency characteristics of fast oscillations.

The source of the oscillation was probably located in AR 6223. On 1990 August 23, the sunspots of AR 6223 were transiting the central meridian and the area of the spot group was approximately its maximum value. The magnetic type was changing into $\beta \gamma$ structure from the normal $\beta$ structure. According to the report of Learmonth Observatory, and others, there was a SF flare located at S08 E10 at 02:53:00 UT. H $\alpha$ flare photographs were also obtained at Yunnan Observatory. Four hours later, once again $\mathrm{H} \alpha$ bright points emerged at the same position. From the Yunnan Observatory $\mathrm{H} \alpha$ photographs, we see that the SF flare consists of two small bright points and one of them is located at the edge of the spot group. An arch-like bridge connects the two bright points. The radio oscillation occurred at just this time.

Figure 1 shows time profiles of the coronal oscillation at $1 \mathrm{MHz}$ frequency increments starting at 07:04:06 UT. From 287 to $289 \mathrm{MHz}$ the oscillations are of small amplitude on top of the smoothly varying background radiation. Above $290 \mathrm{MHz}$ the oscillations are superimposed on a half sine wave which lasts for about $500 \mathrm{~ms}$. The frequency range of the oscillation is 287 to $297 \mathrm{MHz}$, for a 


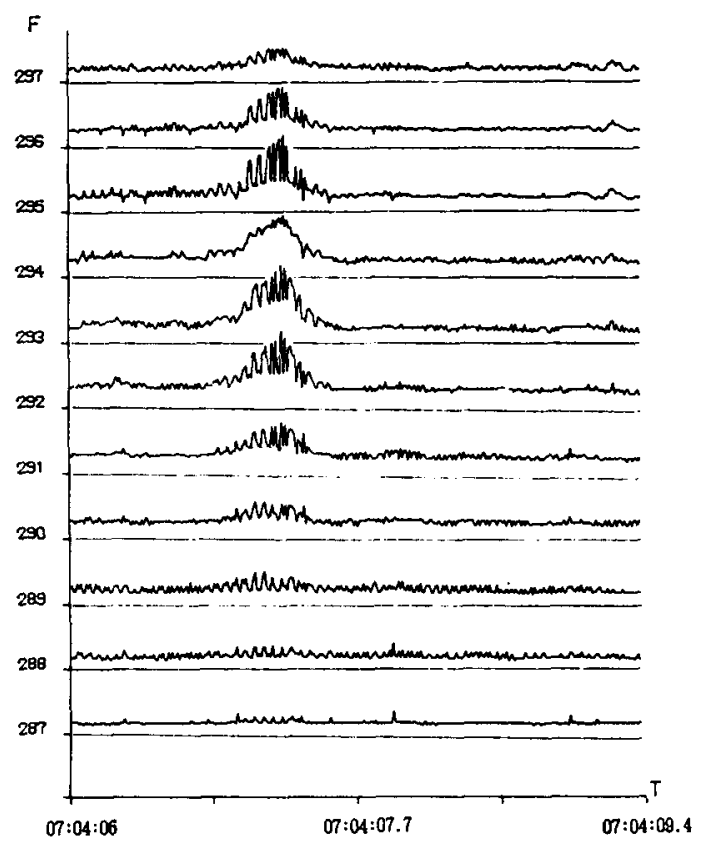

Fig. 1. The coronal oscillation at a series of frequencies.

bandwidth of $10 \mathrm{MHz}$, or a relative bandwidth of $3.4 \%$. The peaks and troughs of the oscillation are clear, so it is easy to count its periods which range from 43 to $108 \mathrm{~ms}$.

This small burst was independently observed at 07:04:07 and $315 \mathrm{MHz}$ on a radio spectrogram obtained at Weissenau, Germany. The time and frequency agreement between the Weissenau observation and ours demonstrates that the radio burst and oscillations observed at Yunnan Observatory are solar in origin. Weissenau Observatory classifies this burst as a spike in Solar Geophysical Data. Details of the radio burst were observed by Yunnan Observatory and Weissenau Observatory and are in good agreement. In the lower frequency band, from 07:03:00 to 07:06:00 UT, there are compact type III radio burst groups among which are single type III radio bursts having very short durations. From 07:05:00 to 07:05:48 UT there were 12 periodic fringes with a period of about $4 \mathrm{~s}$ and a bandwidth that gradually narrowed from 100 to $10 \mathrm{MHz}$. It seems that there is an oscillation with a long period after the spike. The relationships between the $4 \mathrm{~s}$ and $10 \mathrm{~ms}$ oscillations, and the type III bursts will be further studied.

Table 1 contains the radio flux reported by some single frequency observational stations at the time of the oscillations (07:04:00 UT). The radio flux was strong at lower frequencies and cut off at about $950 \mathrm{MHz}$. 
TABLE I Burst flux vs. frequency

\begin{tabular}{ccr}
\hline $\begin{array}{c}\text { frequency } \\
\text { (MHz) }\end{array}$ & $\begin{array}{c}\text { time of maximum } \\
\text { (U.T.) }\end{array}$ & $\begin{array}{c}\text { flux } \\
\text { (s.f.u.) }\end{array}$ \\
\hline 100 & $07: 04: 12$ & 3800 \\
127 & $07: 04: 36$ & 1600 \\
245 & $07: 04: 00$ & 240 \\
410 & $07: 03: 00$ & 60 \\
650 & $07: 04: 18$ & 13 \\
950 & $07: 03: 42$ & 4 \\
\hline \hline
\end{tabular}

\section{RESULTS AND DISCUSSION}

1) At 1990 August 23 07:04:00 UT, Yunnan Observatory and Weissenau observed the radio burst at the same time with solar radio spectrometers. The identity in time and frequency range confirms that the 287 to $300 \mathrm{MHz}$ radio burst recorded at 07:04:06 UT at Yunnan Observatory came from the Sun. The spectrum recorded at Weissenau covers a wide band with moderate resolution and can be used for analyzing general characteristics of the radio burst. Our high-resolution, narrow-band radio spectrometer recording can be used for fine-structure analysis. The fine structures discussed in this paper are periodchanging oscillations with periods of the order of $10 \mathrm{~ms}$.

2) It is convenient to draw flux-frequency spectrum curves and analyze the time evolution of the spectrum curves using our observational data. Figure 2 is the spectrum evolution associated with the oscillations. Each upward step corresponds to a time delay of $10 \mathrm{~ms}$. The oscillations display a drift of radio flux in frequency with a frequency drift of the peak flux of $3.5 \mathrm{MHz}$.

3) Since the 1970s, astronomers and mathematicians like McLean \& Sheridan (1973), Edwin and Roberts (1983), Aschwanden (1987), Pick \& Trottet (1978), Benz (1978), and others have been making theoretical and observational investigations of coronal oscillations. B. Roberts (1984) gave a comprehensive analysis and review of the topic and obtained a series of formulae for oscillation periods. For non-symmetric oscillations (fast kink wave which is similar to a seismic wave) the period can be expressed as

$$
\tau_{f}=\frac{4 \pi^{1 / 2} L}{j}\left(\frac{\rho_{o}+\rho_{e}}{B_{o}^{2}+B_{e}^{2}}\right)^{1 / 2},
$$

and for symmetric oscillations (fast sausage wave which is similar to a marine wave) we have

$$
\tau_{f}^{\prime}=4 \pi^{3 / 2} a\left(\frac{\rho_{o}+\rho_{e}}{B_{o}^{2}+B_{e}^{2}}\right)^{1 / 2},
$$

where $a$ is the radius of the magnetic tube, $L$ is the length of the tube, $\rho_{o}$ and $\rho_{e}$ are the internal and external electron densities of the tube, and $B_{\circ}$ and $B_{e}$ are the internal and external magnetic field intensities of the tube. For fast oscillations of the order of $10 \mathrm{~ms}$, the variations of the period probably depend on variations of the magnetic field intensity of the tube. According to the formulae deduced 


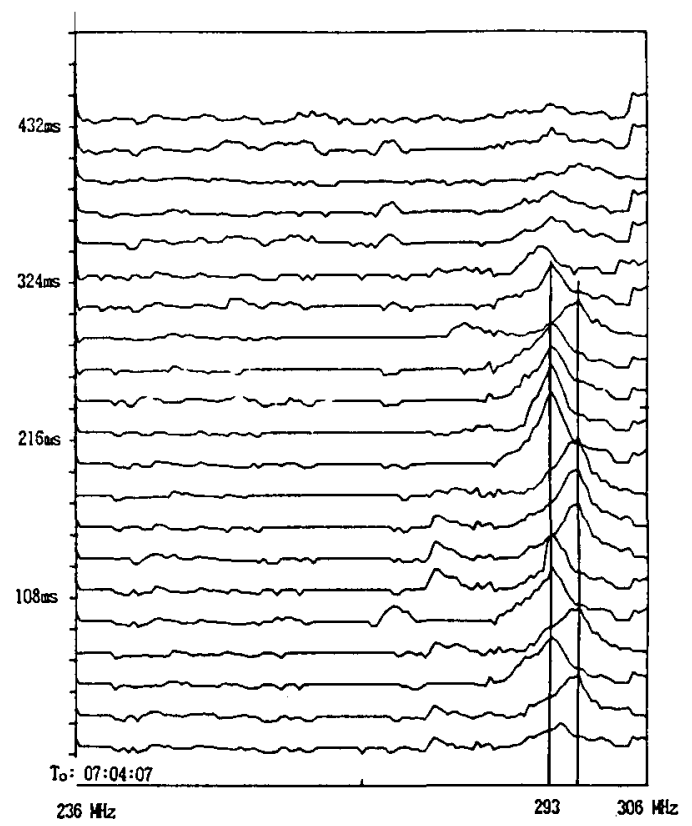

Fig. 2. Evolution of the spectrum.

by $\mathrm{B}$. Roberts, a 2.5 second oscillation period corresponds to oscillations of a magnetic tube with a diameter of $2000 \mathrm{~km}$ and $10 \mathrm{~ms}$ oscillation periods to a micro-magnetic tube with a diameter of $100 \mathrm{~km}$.

4) The spectrum observed at Weissenau shows 12 oscillations with a $4 \mathrm{~s}$ period in the lower frequency range. The period was constant during these oscillations, but the frequency range decreased. This corresponds to an oscillation of a magnetic loop with a diameter of several thousand kilometers.

\section{ACKNOWLEDGMENTS}

The authors thank H. W. Urbarz (Weissenau, Germany) for sharing his beautiful spectrum and $\mathrm{K}$. Ma (Yunnan Observatory, China) for the $\mathrm{H} \alpha$ photographs.

\section{REFERENCES}

Aschwanden, M.J. 1987, Solar Phys., 111, 113

Benz, A. 1978, A\&A, 65, 369

Edwin, P.M. \& Roberts, B. 1983, Solar Phys., 88, 179

McLean, D.J. \& Sheridan, K.V. 1973, Solar Phys., 32, 485

Pick, M. \& Trottet, G. 1978, Solar Phys., 60, 353

Roberts, B. 1984, ApJ, 279, 857 